\title{
CORRECTION
}

\section{Correction to: Infographic of the European Glaucoma Prevention Study (EGPS)}

Ji Eun Han D, Rashmi G Mathew and Christin Henein (D)

(c) The Author(s), under exclusive licence to The Royal College of Ophthalmologists 2021

Eye (2022) 36:903; https://doi.org/10.1038/s41433-021-01713-9

Correction to: Eye https://doi.org/10.1038/s41433-021-01420-5.

The original article has been corrected.

Unfortunately, the middle initial was missing by author's name

Rashmi G. Mathew 